\title{
Evaluation of structural change and local strain distribution in polymers comparatively imaged by FFSA and OCT techniques
}

\author{
E. Leiss-Holzinger ${ }^{*}$, U. D. Cakmak ${ }^{2}$, B. Heise ${ }^{3,4}$, J.-L. Bouchot ${ }^{3}$, E. P. Klement ${ }^{3}$, M. Leitner ${ }^{1}$, \\ D. Stifter ${ }^{4}$, Z. Major ${ }^{2}$ \\ ${ }^{1}$ RECENDT, Research Center for Non Destructive Testing GmbH, Science Park 2/2.OG, Altenberger Straße 69, 4040 \\ Linz, Austria \\ ${ }^{2}$ Institute of Polymer Product Engineering, Johannes Kepler University Linz, Altenbergerstrasse 69, 4040 Linz, Austria \\ ${ }^{3}$ Christian Doppler Laboratory for Microscopic and Spectroscopic Material Characterization, Department of Knowledge- \\ Based Mathematical Systems (FLLL), Johannes Kepler University Linz, Altenbergerstr. 69, 4040 Linz, Austria \\ ${ }^{4}$ Christian Doppler Laboratory for Microscopic and Spectroscopic Material Characterization, Center for Surface- and \\ Nanoanalytics (ZONA), Johannes Kepler University Linz, Altenbergerstr. 69, 4040 Linz, Austria
}

Received 23 June 2011; accepted in revised form 5 October 2011

\begin{abstract}
Mechanical material testing combined with optical coherence tomography (OCT) allows for the first time the immediate detection of inner structural changes along with a qualitative observation of the local strain distribution in surface near bulk regions of semitransparent and translucent specimens. In addition to a 3D full field strain analysis (FFSA) system based on digital image correlation (DIC), a customized spectral domain OCT system operating at $1550 \mathrm{~nm}$ was applied for investigation. Exemplified by tensile testing of elastomer particle filled polypropylene specimens, local dissimilarity evaluation of the OCT images was performed. The results show the high potential of OCT to provide complementary information to DIC-based FFSA, like to identify processes influencing the remaining life of advanced commodity plastics such as the start and progress of yielding, identification of the yielding point, localization of the necking front and the development of small scale voids as in the case of matrix crazing.
\end{abstract}

Keywords: material testing, polymer blends and alloys, damage mechanism, full field strain analysis, optical coherence tomography

\section{Introduction}

The high demands on tailor made materials such as light weight but stiff construction, in addition to aesthetic and haptic properties, are increasingly met by advanced commodity plastics. Various fillers and fibers with different particle size, shape, and surface properties are applied to modify the material's stiffness, strength, and tribological behavior. These are the visible improvements on the macroscopic scale. However, on a mesoscopic scale the material is multiphase and at the corresponding interfaces the material is prone to various defects, as e.g. rupture of elastomer particles resulting in voids [1]. One challenge is to characterize these defects in engineering structures before and during the service in order to visualize the actual mechanical state (stress and strain), with an emphasis on the remaining product life assessment. In the literature and in practice many non-destructive testing and evaluation (NDTE) methods have been introduced with discussions regarding their limitations [2-6].

\footnotetext{
${ }^{*}$ Corresponding author, e-mail: elisabeth.leiss@recendt.at
} (c) BME-PT 
Optical coherence tomography (OCT) is a nondestructive and contactless method for high-resolution imaging of internal structures within turbid media. Invented in the early 90's [7], OCT was originally developed for biomedical applications and is nowadays a well established tool for in vivo diagnostics of eye diseases [8]. In the materials sector, the applications range from imaging of subsurface cracks in ceramics or Teflon, to the investigation of thin multi-layer foils, coatings and injection moulded plastic parts [9]. In the subsurface regions of such semi-transparent and translucent media OCT detects microscopic size defects and structures with a spatial resolution in the micrometer range [10]. The penetration depth strongly depends on the attenuation properties of the material (scattering and absorption) in the used wavelength range. OCT uses light in the near-infrared $(800-1500 \mathrm{~nm})$ and therefore composites containing conducting fibers (e.g. carbon) or metal components are not suitable for OCT imaging due to their opacity. However, a customized OCT system is easy to operate, usually requires no sample preparation and the measurement head can be designed specially towards the needs of the application, e.g. as a hand-held detector. In addition, this method allows the immediate observation of a specimen and its inner structure during material testing. OCT has lately also shown high potential as a high resolution imaging method for in-situ monitoring during arbitrary mechanical loading of heterogeneous polymer structures [11]. Based on the principle of standard photoelasticity [12], the so called polarization sensitive (PS-) OCT [13] technique can yield depth resolved polarization patterns. This method was proven to be applicable for the non destructive quantification of internal stress in polymer materials $[14,15]$, including dynamic measurements during tensile tests [11], but shows two major restrictions: First, samples becoming strongly depolarizing under stress will not show any polarization patterns, as it is the case for the specimens presented in this work. Secondly, a PS-OCT setup detects only stress induced birefringence perpendicular to the incident light beam, which leads to a systematic error. A more promising approach is speckle tracking. Since its invention in the 80 's [16], digital image correlation (DIC) in combination with speckle tracking is used to determine the local strain distribution on surfaces during mechan- ical loading. In order to receive adequate speckles, it is common practice to apply a stochastic color spray pattern on the specimen surface. In contrast, the depth resolved, OCT based method relies on intrinsic speckles inside the real specimen that do not only represent material related information but also noise [17]. In addition, rigid-sample displacement occurring during the testing procedure and several imaging artifacts, like strong reflections at defects, misalignments, or decreasing signal-tonoise ratio over depth, require extended image enhancement. Thus, existing DIC software does not directly work on the recorded OCT images.

For this work tensile tests were performed on elastomer particle filled PP polymer test bars. The local strain on the surface was monitored with a stereoscopic full field strain analysis (FFSA) system based on DIC. As complementary method depth resolved measurements with an OCT system followed by advanced data processing were performed and the promising results are presented in the following.

\section{Experimental setup and method}

Based on white light interferometry, OCT employs light in the near infrared spectral region $[8,18]$. The light coming from the optical source is split into a sample and a reference interferometer arm, with the latter one being terminated by a mirror. The light back reflected from the reference mirror and the different sample structures is then recombined. The so called spectral domain (SD-) OCT uses a broadband light source with the spectrum being detected as a whole by the grating spectrometer, as depicted in Figure 1a). The lateral $(\Delta x)$ and depth resolution $(\Delta z)$ are independent and limited by the optics and the square of the center wavelength $\lambda_{\mathrm{c}}$ over the source bandwidth $(\Delta \lambda)$ (with $\Delta z \propto\left(\lambda_{\mathrm{c}}\right)^{2} /(\Delta \lambda)$ ), respectively [19]. Thus, best resolution is achieved for high bandwidth sources centered at shorter wavelengths. On the other hand, the penetration depth is limited by the attenuation properties in the wavelength range used. It was shown that for most polymers measurements applying $1550 \mathrm{~nm}$ center wavelength resulted in a more than doubled penetration depth when compared to measurements performed at $800 \mathrm{~nm}$ [9]. Thus, the SD-OCT system was equipped with a superluminescence diode centered around $1550 \mathrm{~nm}$ and a spectral width of $55 \mathrm{~nm}$ (Exalos, 


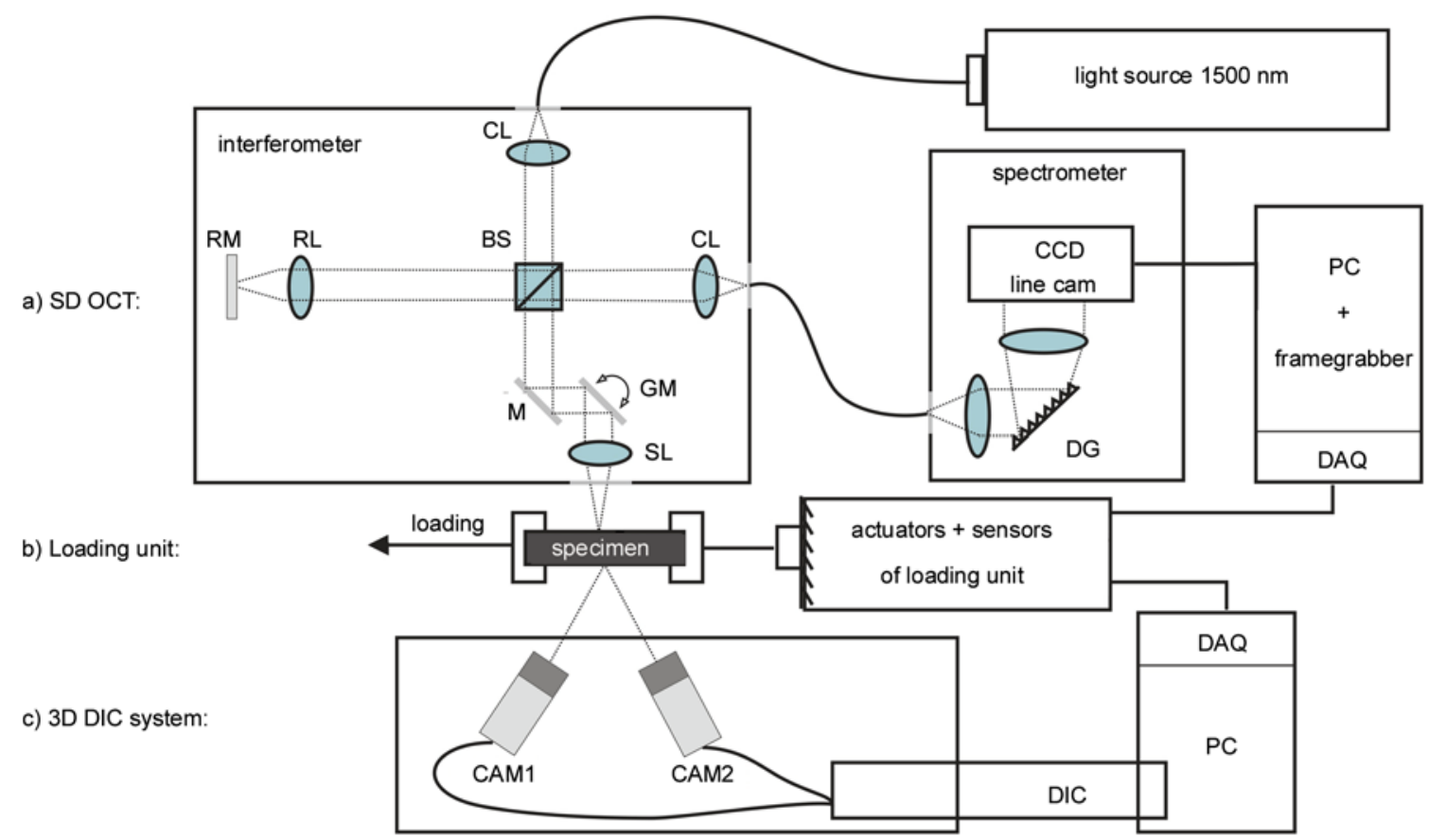

Figure 1. Schematic illustration of the a) spectral domain (SD)-OCT set-up, b) the loading unit and c) the 3D DIC system. Mirror (M), reference mirror (RM), beam splitter (BS), collimator lens (CL), sample lens (SL), reference lens (RL), galvanometer mirror (GM), diffractive grating (DG), data acquisition (DAQ), digital image correlation (DIC).

Switzerland), as well as an InGaAs line camera for the spectrometer. This results in a depth resolution of $19 \mu \mathrm{m}$ in air, which corresponds roughly to $13 \mu \mathrm{m}$ in typical polymer materials with a refractive index $n \sim 1.45$. The interferometer of the OCT setup was built in a Michelson configuration, as shown in Figure 1a. A galvanometer mirror was used to scan the specimen laterally at a lateral resolution of $\sim 14 \mu \mathrm{m}$. Cross sectional images $(1000 \times 512$ pixels $)$ with a sensitivity of $95 \mathrm{~dB}$ over depth were acquired at a frame rate of $4 \mathrm{~Hz}$. A customized compact measurement head allowed the integration into the tensile testing machine (TestBench; Bose Inc; Michigan, USA) that is depicted in Figure 1b. The full field strain analysis based on DIC (ARAMIS, GOM Braunschweig, Germany) was performed by the stereoscopic system depicted in Figure 1c. As samples, specimens of elastomeric particle filled polypropylene (PP) were investigated. The size of the specimens was $35 \times 10 \times 2 \mathrm{~mm}^{3}$. Those specimens were cut from an injection molded flat plaque with a bench shear. Under identical testing conditions, the materials were investigated during loading of the tensile machine in situ with DIC and then with the OCT-setup. To achieve a high temporal resolution considering an OCT frame rate of $4 \mathrm{~Hz}$, the tensile tests were performed at a velocity of $0.01 \mathrm{~mm} / \mathrm{s}$. The maximum displacement was $6.5 \mathrm{~mm}$. Eight specimens were investigated and in both experiments they all showed necking similar in range and position without fracture, with OCT and FFSA providing comparable results.

While the FFSA system surveyed the sample's upper surface in full length ( $x y$-plane in Figure 2a), the OCT images showed a cross sectional area of $9 \mathrm{~mm}$ in length and $3.5 \mathrm{~mm}$ in depth ( $x z$-plane in Figure $2 \mathrm{a}$ ). Figures $2 \mathrm{~b}$ and $2 \mathrm{c}$ show two consecutive OCT frames of the performed measurements. The sample surface was slightly tilted with respect to the sample lens. Due to galvanometer scanning, a small difference in the optical path length of the travelling rays causes an inherent distortion at the left and the right side of the OCT images. Thus, the sample surface appears bent, although it is straight. The OCT images are analyzed in detail in the results section. Advanced image processing methods were applied to determine the local (dis)similarity between temporally subsequent OCT frames, resulting in a so-called Local Dissimilarity (LD-) map, as shown in Figure 2d. Considering a local neighborhood the value of the dissimilarity between subsequent frames was estimated within the corresponding local 


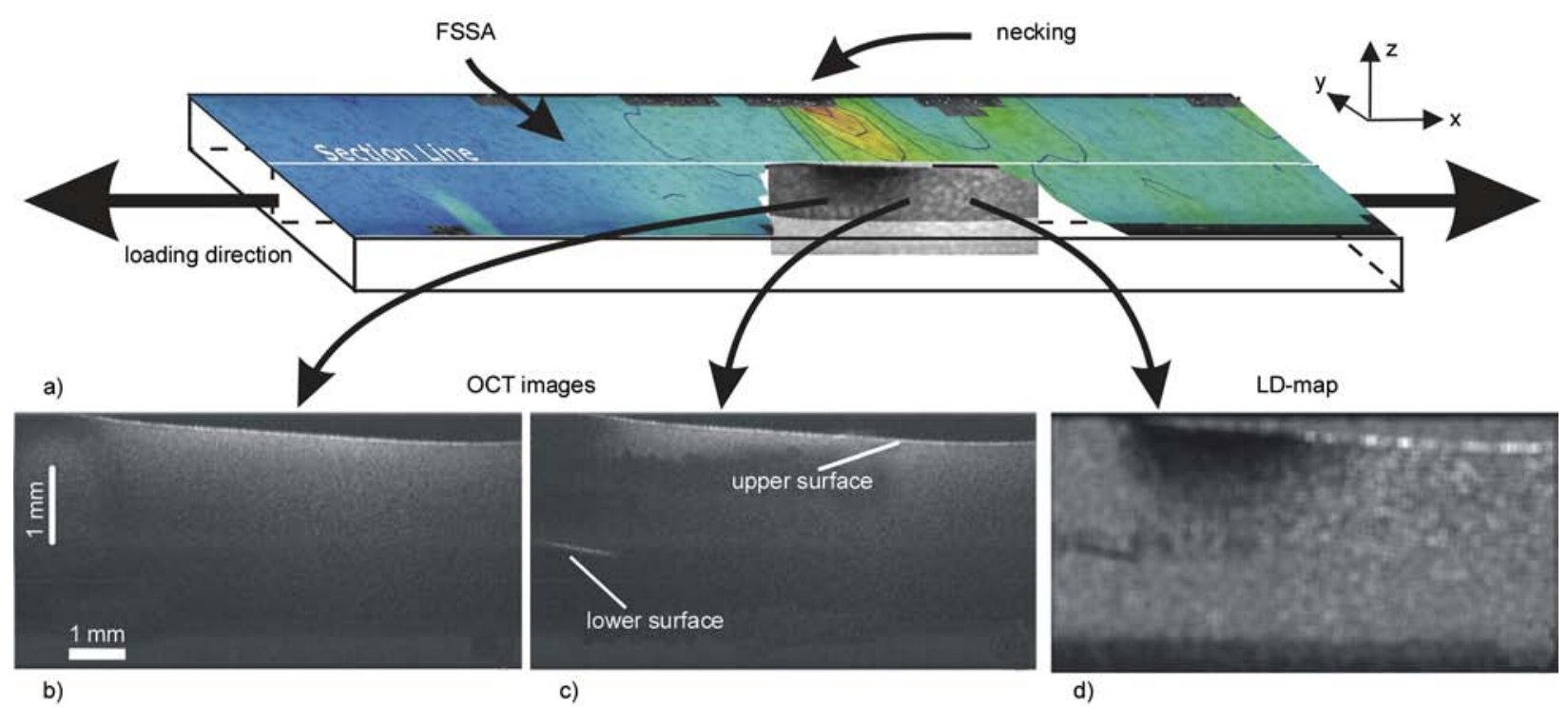

Figure 2. a) Scheme of specimen loaded in axial direction: stress-strain distribution of the upper surface measured with the FFSA system during testing; b) and c) are consecutive OCT scans acquired during testing $(1000 \times 512$ pixels $)$; d) corresponding LD-map

regions. By investigating different dissimilarity measures (be referred to [20]) the Euclidian distance (L2-norm) was chosen here as dissimilarity measure, as depicted in Figure 2d.

\section{Results and discussion}

The global stress-strain curves were derived from the linear variable differential transformer (LVDT) integrated in the loading test bench and they showed good agreement during the measurements via the OCT and the FFSA system, as shown in Figure 3a. Four significant states $i$ to iv were chosen for com-

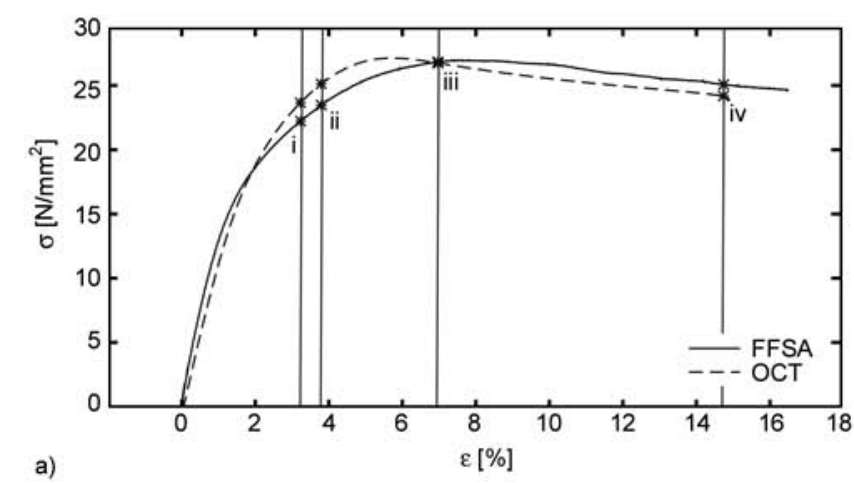

parison of the results, as presented in Figures 3 and 4 . Figure $3 \mathrm{~b}$ shows the color-coded local strain distribution as detected by the FFSA system over the entire upper surface of the specimen. The panels presented in Figure $4 \mathrm{~b}$ only show the region of the OCT measurements and have a lateral extension of just $9 \mathrm{~mm}$. The panels presented in Figure $4 \mathrm{~b}$ correspond therefore to the cross sectional OCT images and the corresponding LD maps shown in Figure $4 \mathrm{c}$ and $4 \mathrm{~d}$. At the beginning of plastic deformation - states $i$ and ii - the global strain is uniformly distributed according to the FFSA measurements shown in Fig- ib)
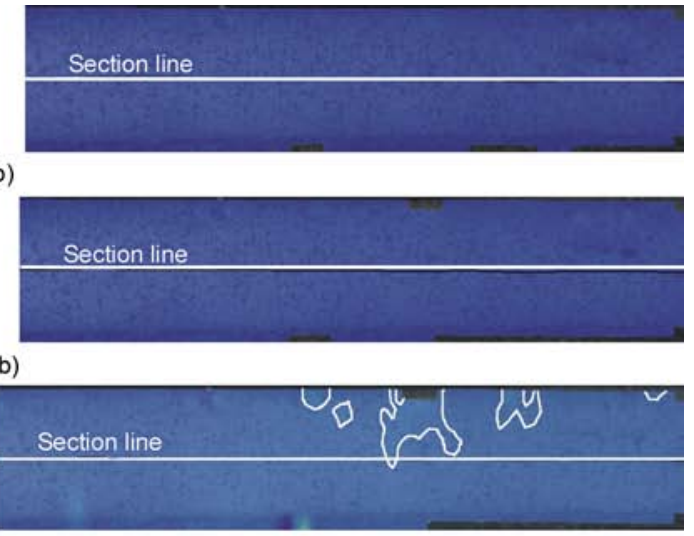

iii b)

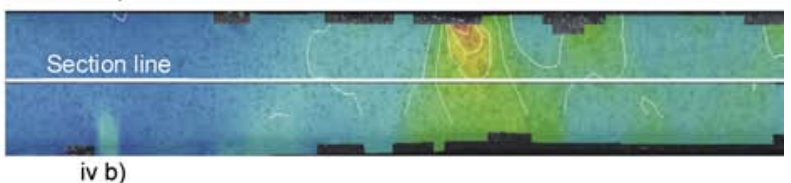
0.0 0.0

Figure 3. a) Global stress-strain curves measured during the OCT and the FFSA measurement via a linear variable differential transformer (LVDT); b) local strain measured by the FFSA system; the states i to iv are analyzed in detail in the results section 


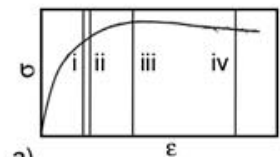

a)

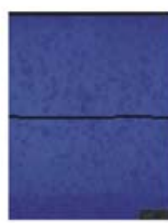

i b)

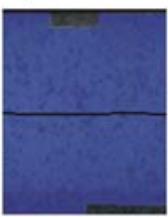

ii b)

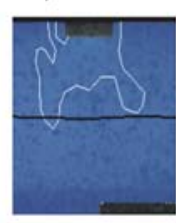

iii b)

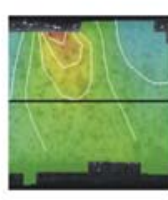

iv b)

b) FFSA

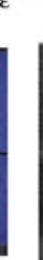

i c)

ii c)

iii c)

iv c)

c) OCT
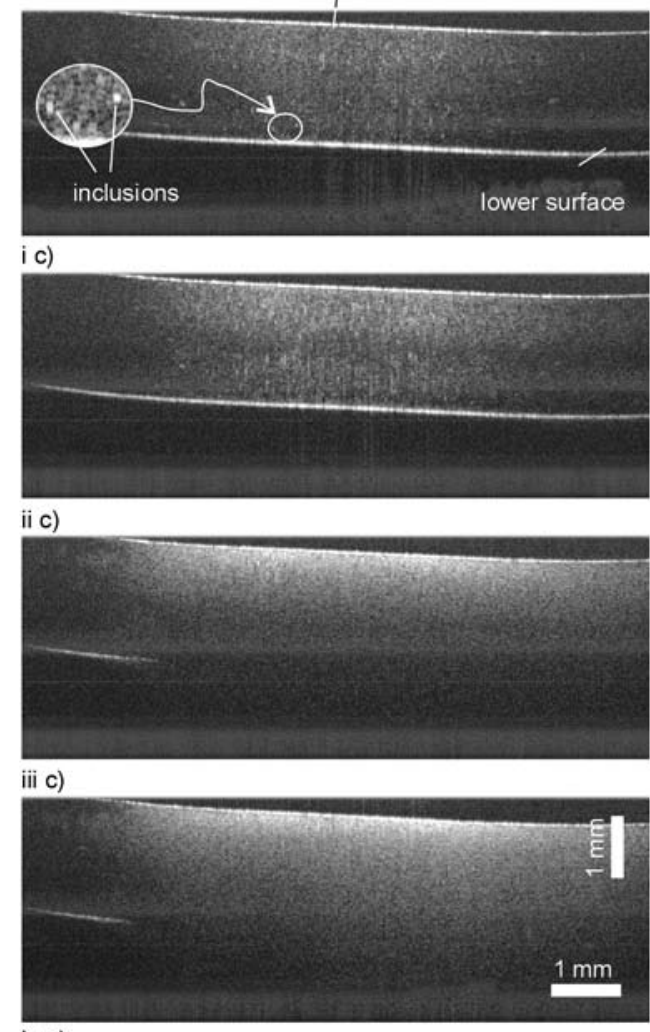

0.0

local $\varepsilon_{x}[\%]$

60.0

$\begin{array}{lll}15.0 & 30.0 & 45.0\end{array}$

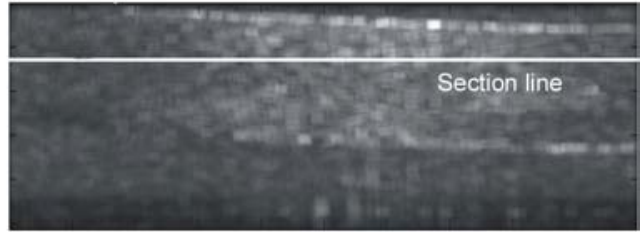

id)
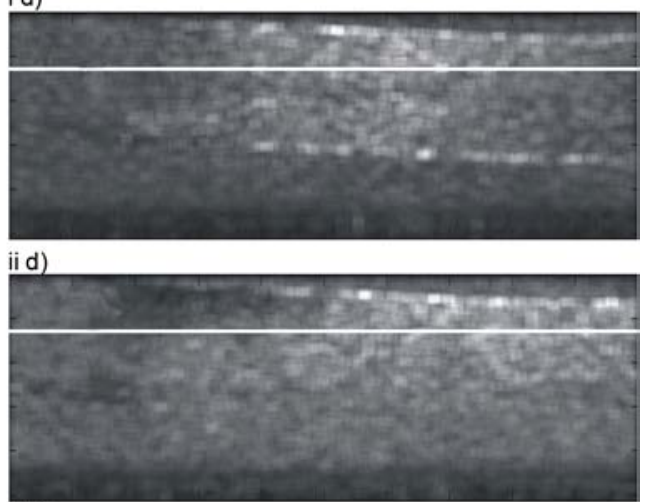

iii d)

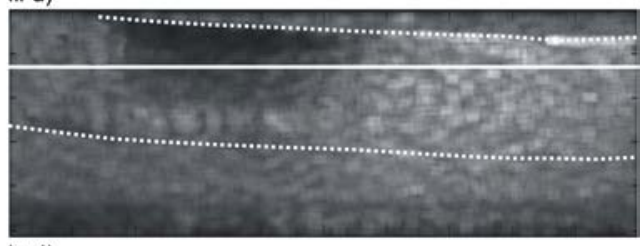

iv d)

d) LD-maps

Figure 4. a) Global stress-strain curve; b) local strain measured by the FFSA system; c) OCT measurement within the specimen's cross-section; d) corresponding local dissimilarity map

ure 3 and as magnified detail in Figure $4 \mathrm{ib}$ and 4 iib. In contrast, the OCT image already reveals a change in the material properties: in Figure 4 ic the specimen is still highly translucent showing air inclusions around agglomerated elastomer particles, whereas in the next state Figure 4 iic, the development of a brighter region near the upper surface indicating increasing scattering of the material shows up. This can be attributed to small-scale cavitations between the elastomer particles and the host matrix [20]. The corresponding LD maps in Figures $4 \mathrm{id}$ and 4 iid show a bright region in the center, reflecting this beginning change in opacity. The remaining darker region, including the air beyond the lower surface, is a measure for the high similarity between consecutive frames, and thus also reflects the high signal-to-noise ratio. In states iii and iv flowing of the material is observed: the FFSA measurement shows the onset of necking in Figure 3 and as mag- nified detail in Figure 4 iiib and an advanced state in Figures 3 and 4 ivb. The brightness of the specimen in the OCT images 4 iiic and 4 ivc indicates high scattering of the material implying the beginning of matrix crazing and stress whitening. Multiply scattered photons lead to a reduced penetration depth of OCT and so the lower surface is no more visible. The lower half of the OCT image is dominated by noise of random nature, leading to dissimilarity between consecutive frames. This corresponds to the bright regions in the lower half of the LD maps in Figures 4 iiid and 4 ivd. Near the upper surface two regions can clearly be distinguished: an increasing dark area representing a static region within the specimen, and to the right a bright region indicating the flowing of material. These two regions form a border, which can be clearly observed and represents, from the microscopic point of view the interface between the static and the highly dynamic area, 
and in the macroscopic view the front of the necking.

For better comparison with the global stress strain curve, a characteristic value representing the whole LD-map was needed. Anyhow, the lower part of the LD-map was not taken into account, since it includes a large area of air under the sample and shows significant noise in case of reduced penetration depth (state iii, iv). Thus, only the upper half of the image was considered as region of interest, as shown in Figure 5a. As characteristic value, we calculated the percentage of the total 'static area' with respect to the total area in the LD-map. A static area is an area of high similarity between consecutive frames and is represented by dark pixels. In contrary, a dynamic area is an area of low similarity between consecutive frames and is represented by bright pixels. Considering that not only material yielding but also overall elongation, rigid sample movements and noise 'brighten' a pixel, the determination of an appropriate gray level as limit between static and dynamic regions is not trivial. Two alternative grey levels were found suitable for further processing; the first one was chosen near the lower knee of the histogram as shown in Figure 5a. Regions brighter than this level were assumed to be highly dynamic. Regarding the second gray level, regions darker

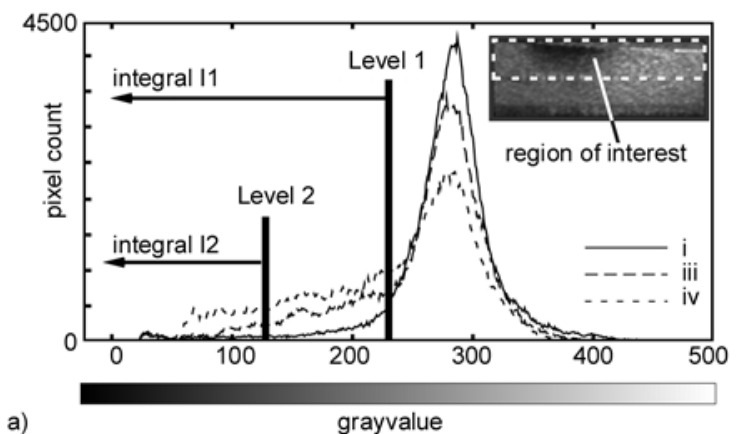

grayvalue

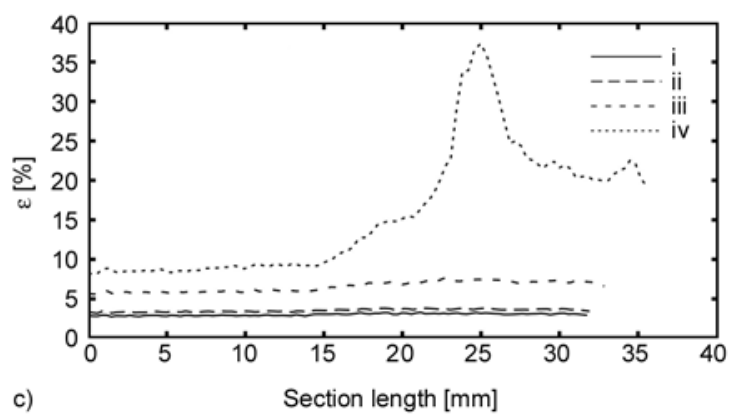

than this level were assumed to be truly static. For calculation of the characteristic value, pixels with values lower than the respective gray level were summed-up via integration of the histogram (I1, I2), as depicted in Figure 5a. To visualize the continuous development of necking within the sample, the resulting curves, I1 and I2, were compared to the global stress strain curve in Figure 5b: The beginning of the tensile test is dominated by a lack of similarity due to rigid movements of the specimen followed by its overall elongation. Thus, the percentage of the static area remains at its minimum for both variants, I1 and I2. At state i a first significant increase of I1 is observed, reflecting a considerable reduction of the dynamic area, and indicating that the specimen has reached a stable position. I 2 does not increase until the formation of a static region within the specimen, indicated by an arrow in Figure $5 b$. This coincides with the moment when crossing the yield point and marks the beginning of the non-uniform elongation flow.

To illustrate the local development of the overall elongation Figure $5 c$ depicts the strain measured along the white section lines indicated in Figure $3 \mathrm{~b}$. For state iv the local strain peaks due to necking. Figure $5 \mathrm{~d}$ shows a comparison of the gray values obtained along the white section lines drawn in Fig-

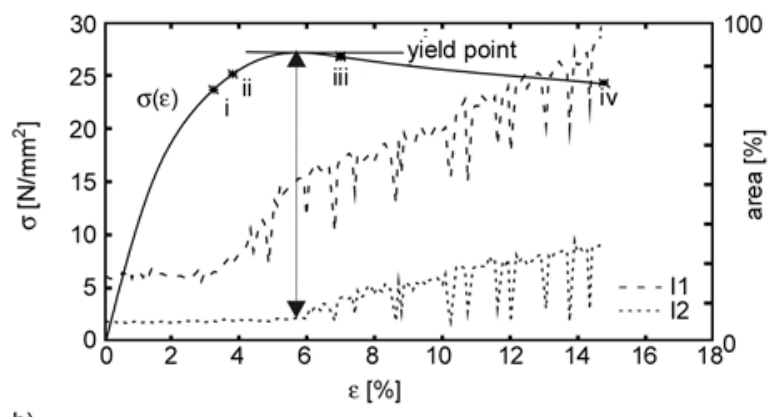

b)

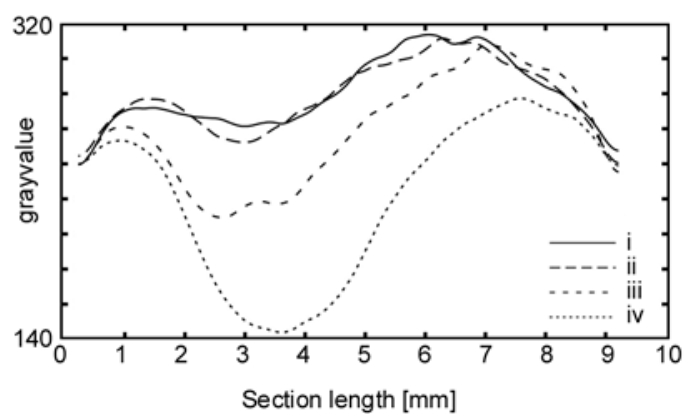

Figure 5. a) LD-Map histograms for three states (i, ii, iii) based on the upper half of the LD-Map (region of interest). b) OCT: Global stress strain curve (left y-scale) in comparison with characteristic values I1 and I2 (right y-scale) computed from the LD-Map. c) FFSA: Local distribution of strain along the section line given in Figure 3b; d) OCT: Local distribution of gray value along the section line of Figure 4d. 
ure $4 \mathrm{~d}$. The curves show an increasing slope, which corresponds to a rising non-uniformity in elongation.

\section{Conclusions}

As demonstrated in this work, a combined evaluation approach by FFSA and OCT provides novel complementary information and thus represents a promising procedure to investigate in situ dynamic processes occurring inside scattering specimens during tensile tests. In addition to observing the development of micro defects and the progression of structural change, the depth resolved necking front between static and flowing material was detected. OCT is applicable to specimens of arbitrary size, affords no extensive radiation protection in contrast to X-ray computed tomography (CT), and can be customized to be integrated in a testing device. Furthermore, by means of speckle tracking the local distribution of strain can be determined and turns OCT superior to conventional techniques like standard photoelasticity.

Consequently, this great potential is now to be exploited through (i) an enhancement of the OCT setup with respect to higher penetration depth and resolution in both space and time, (ii) a systematic collection of measurement data derived simultaneously via OCT and FFSA systems, and (iii) advanced data processing.

Beyond the qualitative material characterization given already by the local dissimilarity map, furthermore, an extended data processing is needed for analyzing the OCT-imaged material behavior in a quantitative way. A combined statistical and correlation based multi-scale analysis of the speckle patterns is suggested to distinguish between noise and information carrying speckles and finally to calculate the displacement and strain fields. In addition, information in the LD-maps is found primarily in the characteristics of the local distribution. Thus, a more sophisticated approach than the evaluation of the static area portion is needed to generate a scalar quantity describing the whole LD-map or the stress/ strain map. Finally, advanced data processing can reveal significant processes occurring within the specimen, such as the start and progress of yielding, identification of the yielding point, localization of the necking front and the development of small scale voids as in the case of matrix crazing.

\section{Acknowledgements}

We thank C.K. Hitzenberger and his group at the Medical University of Vienna for continuous advice in OCT technology. The financial support by the Austrian Science Fund (project P19751-N20), the European Regional Development Fund in the framework of the EU-programme Regio 13, the federal state Upper Austria, the Austrian Federal Ministry of Economy, Family and Youth and the National Foundation for Research, Technology and Development is gratefully acknowledged.

\section{References}

[1] Karaeva A. A., Serenko O. A., Goncharuk G. P., Bazhenov S. L.: New mechanism of failure of a dispersion-filled polymer composite. Physical Chemistry, 423, 289-291 (2008).

DOI: $10.1134 / \mathrm{S} 0012501608110018$

[2] Bohse J., Kroh G., Wolf H.: Acoustic emission characterizes micro mechanic behavior (in German). Kunststoffe, 81, 543-547 (1991).

[3] Bar-Cohen Y.: Emerging NDE technologies and challenges at the beginning of the $3^{\text {rd }}$ milleneum - Part I. Material Evaluation, 58, 17-30 (2000).

[4] Bar-Cohen Y.: Emerging NDE Technologies and challenges at the beginning of the $3^{\text {rd }}$ milleneum - Part II. Material Evaluation, 58, 141-150 (2000).

[5] Predak S., Lütze S., Zweschper T., Stößel R., Busse G.: Comparative non-destructive characterization. Damage of short glass fiber reinforced polypropylene (in German). MP Materials Testing, 43, 14-18 (2002).

[6] Hendorfer G.: Active thermography for quantitative analysis of defects (in German). MP Materials Testing, 51, 400-404 (2009)

[7] Huang D., Swanson E. A., Lin C. P., Schuman J. S., Stinson W. G., Chang W., Hee M. R., Flotte T., Gregory K., Puliafito C. A., Fujimoto J. G.: Optical coherence tomography. Science, 254, 1178-1181 (1991). DOI: $10.1126 /$ science.1957169

[8] Bouma B. E., Tearney G. E.: Handbook of optical coherence tomography. Marcel Dekker, New York (2002).

[9] Stifter D.: Beyond biomedicine: A review of alternative applications and developments for optical coherence tomography. Applied Physics B: Lasers and Optics, 88, 337-357 (2007). DOI: $10.1007 / \mathrm{s} 00340-007-2743-2$

[10] Stifter D., Wiesauer K., Wurm M., Schlotthauer E., Kastner J., Pircher M., Götzinger E., Hitzenberger C. $\mathrm{K}$.: Investigation of polymer and polymer/fibre composite materials with optical coherence tomography. Measurement Science and Technology, 19, 074011/1074011/8 (2008). DOI: $10.1088 / 0957-0233 / 19 / 7 / 074011$ 
[11] Stifter D., Leiss-Holzinger E., Major Z., Baumann B., Pircher M., Götzinger E., Hitzenberger C. K., Heise B.: Dynamic optical studies in materials testing with spectral-domain polarization-sensitive optical coherence tomography. Optics Express, 18, 25712-25725 (2010). DOI: $10.1364 /$ OE.18.025712

[12] Ramesh K.: Digital photoelasticity: Advanced techniques and applications. Springer, Heidelberg (2000).

[13] de Boer J. F., Milner T. E., van Gemert M. J. C., Stuart Nelson J.: Two-dimensional birefringence imaging in biological tissue by polarization-sensitive optical coherence tomography. Optics Letters, 22, 934-936 (1997).

DOI: 10.1364/OL.22.000934

[14] Wiesauer K., Sanchis Dufau A. D., Götzinger E., Pircher M., Hitzenberger C. K., Stifter D.: Nondestructive quantification of internal stress in polymer materials by polarisation sensitive optical coherence tomography. Acta Materialia 53, 2785-2791 (2005). DOI: $10.1016 /$ j.actamat.2005.02.034

[15] Wiesauer K., Pircher M., Goetzinger E., Hitzenberger C. K., Engelke R., Ahrens G., Gruetzner G., Stifter D.: Transversal ultrahigh-resolution polarizationsensitive optical coherence tomography for strain mapping in materials. Optics Express, 14, 5945-5953 (2006). DOI: $10.1364 / \mathrm{OE} .14 .005945$
[16] Bruck H. A., McNeill S. R., Sutton M. A., Peters W. H.: Digital image correlation using Newton-Raphson method of partial differential correction. Experimental Mechanics, 29, 261-267 (1989).

DOI: $10.1007 / \mathrm{BF} 02321405$

[17] Kirkpatrick S. J., Wang R. K., Duncan D. D.: OCTbased elastography for large and small deformations. Optics Express, 14, 11585-11597 (2006). DOI: 10.1364/OE.14.011585

[18] Sheppard C. J. R., Roy M., Sharma M. D.: Image formation in low-coherence and confocal interference microscopes. Applied Optics, 43, 1493-1502 (2004).

[19] Brezinski M. E.: Optical coherence tomography: Principles and applications. Academic Press, Amsterdam (2006).

[20] Stifter D., Heise B., Bouchot J-L., Major Z., LeissHolzinger E., Pircher M., Götzinger E., Baumann B., Hitzenberger C. K.: Spectral domain polarization sensitive optical coherence tomography at $1.55 \mu \mathrm{m}$ : Novel developments and applications for dynamic studies in materials science. in 'Proceeding of Optical Coherence Tomography and Coherence Domain Optical Methods in Biomedicine XV, San Francisco, USA' $78890 Z$ (2011).

DOI: $10.1117 / 12.873542$ 